\title{
Ordinal Optimization Theory Based Planning for Clustered Wind Farms Considering the Capacity Credit
}

\author{
Yi Wang*, Ning Zhang*, Chongqing Kang ${ }^{\dagger}$, Qianyao Xu*, Hui Li**, Jinyu Xiao**, Zhidong Wang**, \\ Rui Shi** and Shuai Wang**
}

\begin{abstract}
Wind power planning aims to locate and size wind farms optimally. Traditionally, wind power planners tend to choose the wind farms with the richest wind resources to maximize the energy benefit. However, the capacity benefit of wind power should also be considered in large-scale clustered wind farm planning because the correlation among the wind farms exerts an obvious influence on the capacity benefit brought about by the combined wind power. This paper proposes a planning model considering both the energy and the capacity benefit of the wind farms. The capacity benefit is evaluated by the wind power capacity credit. The Ordinal Optimization (OO) Theory, capable of handling problems with non-analytical forms, is applied to address the model. To verify the feasibility and advantages of the model, the proposed model is compared with a widely used genetic algorithm (GA) via a modified IEEE RTS-79 system and the real world case of Ningxia, China. The results show that the diversity of the wind farm enhances the capacity credit of wind power.
\end{abstract}

Keywords: Capacity credit, Correlation, Ordinal optimization, Planning, Wind power

\section{Introduction}

Wind power as a clean and renewable energy resource has shown impressive growth all over the world in the last decade. In China, the total installed wind power capacity reached $91.41 \mathrm{GW}$ by the end of 2013 [1] and is expected to reach $200 \mathrm{GW}$ by the end of 2020 [2]. Most of the rich onshore wind resources of China are concentrated in eight planned $10 \mathrm{GW}$ wind power bases. Each wind power base covers one thousand square kilometers in area and contains several sub-zones available for installing wind farms [3]. An optimal solution to coordinate the planning of wind farms in each sub-zone can be found to maximize the benefit, in both time sequence and space perspective.

The optimization of wind farm planning has been widely researched. $\mathrm{Xu}$ and Zhuan [4] proposed a wind farm planning model to minimizes the power system operation cost, the reserve cost, the value of load shedding and the opportunity cost of wind curtailments. The planning problem is modeled using a chance-constrained programming approach and solved in combination with a Monte Carlo approach, neural network and genetic algorithm. Baringo and Conejo [5] presented a riskconstrained two-stage wind farm planning model, which considered the planning decision in the first stage and the

$\dagger \quad$ Corresponding Author: State Key Lab of Power Systems, Department of Electrical Engineering, Tsinghua University, Beijing, China. (cqkang@tsinghua.edu.cn)

* State Key Lab of Power Systems, Department of Electrical Engineering, Tsinghua University, Beijing, China. (\{wangyi14, ningzhang,xuqy06\}@mails.tsinghua.edu.cn)

** State Power Economic Research Institute, Beijing, China.

Received: December 31, 2014; Accepted: May 22, 2015 market clearing of different wind power output scenarios in the second stage. The model was transformed into a large scale Mixed Integer Linear Programming (MILP) problem and solved using CPLEX. The same method was used in a transmission planning problem considering wind power output [6]. Nick et al. [7] established an optimal wind farm allocation model considering the capacity limits of the transmission networks. The problem is decomposed into a master problem determining wind farm allocation and a sub-problem simulating annual unit commitment accompanied by optimal DC power flow, which is solved with Benders decomposition. Liu et al. [8] proposed a robust optimization planning model to investigate the benefit of wind farm diversification for power system operation and reliability. The model is transformed into a coupled problem composed of a linear programming problem and a conic quadratic programming problem to be solved.

Traditionally, the optimization of the wind farm planning tends to choose the wind farm with the best wind resources to maximize the energy benefit. However, the capacity benefit of wind power should also be considered in the case of large-scale clustered wind farm planning because the clustered wind farms can act as a firm load-carrier and benefit the power system in terms of reducing the investment in traditional plants $[9,10]$. Previous research has shown that diversifying the wind farm could smooth out the fluctuations in wind power generation and improve the load carrying capability of wind power. Lu et al. [11] studied the optimal integration of offshore wind farms in China and showed that installing offshore wind farms in the dispersed regions of Bohai Bay, the Yangtze River 
Delta, and the Pearl River Delta could significantly smooth out the variability of wind power so that $28 \%$ of the overall offshore wind potential could be deployed as base-load power. Placing all the wind farms in some areas with rich resources might not be the best choice because a diversified distribution of wind farms would obtain a higher credit capacity [12, 13].

The extended research of [14] is presented in the paper, which continues focusing on the planning of wind farms while maximizing the energy benefit and capacity benefit. The capacity benefit is measured by the capacity credit for the wind power, which denotes the ratio of equivalent conventional generation capacity that the wind power can exhibit in a power system on a power system adequacy basis. The planning optimization model is proposed and solved using the Ordinal Optimization (OO) Theory.

The rest of the paper is organized as follows: Section 2 first introduces and provides an overview of the concept and methodology of the capacity credit for wind power. The wind power planning model is then presented and solved by the OO-based method in Section 3 and Section 4, respectively. Section 5 verifies the proposed approach by an illustrative example using the modified IEEE RTS79 test system. A case study in Ningxia, China, is proposed using the same approach in Section 6. Finally, the conclusions are drawn in Section 7.

\section{Wind Power Capacity Credit}

\subsection{Definition of wind power capacity credit}

Wind power can carry the load at some firm capacity and thus benefits the power system in terms of refraining from or deferring the investment in conventional power generation. The capacity credit of wind power denotes the ratio of the capacity that wind power could contribute to the supply adequacy of the system compared to a conventional mode of generation, e.g., thermal generation or hydrogeneration. There are various similar definitions of capacity credit [15], among which the definition of Equivalent Firm Capacity (EFC) is the most widely used. Mathematically, capacity credit is defined as the following equation:

$$
R\left\{C_{\mathrm{r}}+C_{\mathrm{v}}\right\}=R\left\{C_{\mathrm{r}}+C_{\mathrm{w}}\right\}
$$

where $C_{\mathrm{r}}, C_{\mathrm{v}}$ and $C_{\mathrm{w}}$ represent the capacity of conventional units, the capacity of fictitious $100 \%$ reliable units, and the capacity of wind farms respectively. $R\{\cdot\}$ stands for the calculation of the reliability index used for adequacy evaluation, e.g. Loss Of Load Expectation (LOLE) or Expected Energy Not Served (EENS). If (1) holds, $C_{\mathrm{v}}$ is defined as the wind power creditable capacity and then the capacity credit $\lambda$ is defined as the ratio of $C_{\mathrm{v}}$ to $C_{\mathrm{w}}$.

$$
\lambda=\frac{C_{\mathrm{v}}}{C_{\mathrm{w}}}
$$

\subsection{Evaluation of wind power capacity credit}

Properly evaluating the wind power capacity credit is challenging because the value of the capacity credit $\lambda$ is affected by various factors. Normally, a better wind resource would result a higher capacity credit. Almost all the studies have shown that the capacity credit of wind power decreases as the wind power penetration increases. The wind farms with more dispersed outputs are found to result in a higher capacity credit than the same capacity of wind farm with highly correlated output [16].

Evaluation methods for capacity credit can be roughly divided into the Monte Carlo approach [17], the convolution approach [18] and the analytical approximation approach [19]. The Monte Carlo approach and convolution approach are more accurate but relatively time-consuming. However, the analytical approximation approach is more efficient but may not be as accurate and can be used only in rough estimations. An analytical form of wind power capacity credit in [18] is used for the evaluation of the wind power credit in this paper:

$$
\begin{aligned}
& \lambda=\sum_{t=1}^{T}\left(\frac{k_{t}}{\sum_{t=1}^{T} k_{t}} \frac{P_{t}}{C_{\mathrm{w}}}\right) \\
& \text { where } k_{t}=\frac{f_{R F}\left(d_{t}-P_{t}+\lambda C_{\mathrm{w}}\right)-f_{R F}\left(d_{t}\right)}{\lambda C_{\mathrm{w}}-P_{t}}
\end{aligned}
$$

where $f_{R F}\left(d_{t}\right)$ is the reliability function with the load of $d_{t}$, and $P_{t}$ represents the output of wind power for hour $t$. Eq. (3) shows that the wind power capacity credit is a linear combination of the wind power output ratio. While $k_{t}$ has the term of $\lambda$ which is not known to be a priority, the analytical form of the wind power capacity credit $\lambda_{a}\left(x_{1, a}, \ldots, x_{N, a}\right)$ is not an explicit expression and requires an evaluation approach.

\section{Modeling of Wind Farm Planning Considering the Capacity Credit}

\subsection{Nomenclature}

$x_{i, a} \quad$ State variable for the investment decision of wind farm $i$ during the year $a$ (equals 1 if the wind farm is installed in the year $a$; equals 0 if the wind farm is installed before or after the year $a$ ).

$F_{i, a}$ Annualized capital cost, operation and maintenance cost of wind farm $i$ during the year $a(\$)$.

$B_{\text {Energy, } i, a}$ Energy benefit of wind farm $i$ during the year $a(\$)$. 
$B_{\text {Capacity, } a}$ Capacity benefit of wind farm $i$ during the year $a(\$)$.

$r \quad$ Interest rate used in calculating the annualized investment cost.

$K_{\mathrm{w}} \quad$ Payback period of wind farm investment.

$K_{\mathrm{c}} \quad$ Payback period of conventional thermal generator.

$(\mathrm{P} / \mathrm{A}, r, K)$ Coefficient of equivalent annual investment with the interest rate $r$ and payback period $K$. $(\mathrm{P} / \mathrm{A}, r, K)=\left[(1+r)^{K}-1\right] / r(1+r)^{K}$

$I_{\mathrm{w}, i} \quad$ Unit investment cost of wind farm $i(\$ / \mathrm{kW})$.

$I_{\mathrm{c}, i} \quad$ Unit investment cost of conventional thermal generator $i(\$ / \mathrm{kW})$.

$O_{i} \quad$ Unit yearly operation and maintenance (O\&M) cost for wind farm $i(\$ / \mathrm{kW})$.

$P_{i, a, t}$ Simulated power output of wind farm $i$ for hour $t$ during the year $a(\$)$.

$\rho_{i} \quad$ Energy price of wind farm $i(\$ / \mathrm{MWh})$.

$C_{\mathrm{w}, i}$ The installed capacity of wind farm $i$ (MW).

$\lambda_{a}\left(x_{1, a}, \ldots, x_{N, a}\right)$ The capacity credit of all the wind farms during the year $a$ under the investment decision of $x_{1, a}, \ldots, x_{N, a}$.

$C_{\text {Plan, } a}$ Requirement of wind power installation for the year $a(\mathrm{MW})$.

$N$ Number of candidate wind farms considered in the planning model.

$Y \quad$ Number of continuous target years considered in the planning model.

\subsection{Assumptions}

In this section, we try to demonstrate how the capacity credit of wind power can be maximized in wind power planning. To discuss this issue more clearly, we simplify the current wind power planning model with three assumptions, as follows:

1) The capacity requirement for wind power is set before the planning. Because the proper capacity of wind power is determined by the power system flexibility, the cost effectiveness of wind power and other policy issues are not the focus of this paper. The constraints related to the operation of the power system are therefore omitted in the model.

2) The information on the wind resources of each candidate wind farm is available before planning. The hourly wind power can be simulated according to the characteristics of a wind resource such as the distribution of wind speeds and the correlation between the wind speeds. The simulated hourly wind power output will be used in the planning model as the basis of calculating the capacity credit of wind power.

3) The planning of generations has been determined, and the hourly load forecasting or simulation result is available because the capacity of other generations and level of load have a great impact on the evaluation of the wind power capacity credit.
The long-term planning of wind power over several years is considered in the model to optimize the investment potential of wind farms. Therefore, the net present value (NPV) of the capacity benefit over all the years studied is considered in the model.

\subsection{Objective function}

The object of the planning is to minimize the net cost (maximize the net benefit) from the social perspective, which includes the capital cost and the O\&M cost of the wind farm, while the benefit includes the energy benefit and the capacity benefit. Both the considered cost and the benefit are associated with the target years in the planning problem. To simplify, the cost and benefit in different years are aggregated as the NPV of the first target years of the planning model.

The capital cost and O\&M cost are annualized considering the interest rate and its payback period.

$$
F_{i, a}=(1+r)^{-a}\left[\left(\mathrm{P} / \mathrm{A}, r, K_{\mathrm{w}}\right) I_{\mathrm{w}, i}+O_{i}\right] C_{\mathrm{w}, i} x_{i, a}
$$

The energy benefit of the wind farm is modeled using the simulated hourly output multiplied by its energy price:

$$
B_{\text {Energy }, i, a}=(1+r)^{-a} \rho \sum_{t=1}^{T} P_{i, a, t} X_{i, a}
$$

The capacity benefit of the wind farm is modeled using the credible capacity of wind power multiplied by the investment of conventional thermal generation:

$$
B_{\text {Capacity }, a}=\left(\mathrm{P} / \mathrm{A}, r, K_{\mathrm{c}}\right) I_{\mathrm{w}, i} \lambda_{a}\left(x_{1, a}, \ldots, x_{N, a}\right) \sum_{i=1}^{N} C_{\mathrm{w}, i} x_{i, a}
$$

Successively, the objective function of the planning model can be written as

$$
\min \sum_{i=1}^{N} \sum_{a=1}^{Y} F_{i, a}-\sum_{i=1}^{N} \sum_{a=1}^{Y} B_{\text {Energy }, i, a}-\sum_{a=1}^{Y} B_{\text {Capacity }, a}
$$

\subsection{Constraints}

The capacity requirement of wind power for each year should be achieved in the target years:

$$
\sum_{i=1}^{N} \sum_{a=1}^{\tilde{a}} x_{i, a} C_{w, i} \geq C_{\text {Plan }, a}
$$

Each wind farm can be invested only once, or not be invested:

$$
\sum_{a=1}^{\tilde{a}} x_{i, a} \leq 1
$$


Other constraints for the wind farm planning model are also available. For example, the earliest installed year of each wind farm can be set as a direct constraint on $x_{i, a}$. The associated power system operational constraints can also be added. However, these constraints can all be constructed in the MILP form and do not change the mathematical characteristics of this problem.

\section{OO Theory Based Approach}

\subsection{OO Theory}

Because the term of capacity credit $\lambda_{a}\left(x_{1, a}, \ldots, x_{N, a}\right)$ is not an explicit expression, the proposed model cannot be solved directly. The OO theory is a novel approach to cope with the complex and difficult optimization problem and can significantly reduce the number of search samples of the huge feasible solution space formed by all discrete control variables [20, 21]. The OO theory aims to obtain sufficiently good solutions with a high probability instead of finding the perfect optimal solutions to avoid time consuming calculation. The OO theory has been widely used in many aspects such as bidding strategy for electric power suppliers [22], optimal power flow with discrete variables [23], transmission system enhancement [24], reactive optimization in distribution systems [25], etc.

The principle of $\mathrm{OO}$ is that the random selection of feasible solutions would cover some sufficiently good solutions, and the crude model evaluation gives a rough ordering of the solutions. If we pick some certain number of solutions properly, we would be confident that the solution selected would include one or more sufficiently good solutions. Because the crude evaluation model is much faster than the accurate evaluation, this approach can improve the overall solving efficiency for the complex problem. OO has been applied to optimization problems in the power system [20]. Fig. 1 shows a visual relationship between the set of solutions defined in the OO approach. The details of this approach can be found in [21].

The procedure of $\mathrm{OO}$ is to first generate a large number of feasible solutions and use the crude model to rapidly evaluate the performance of each solution. Then, the

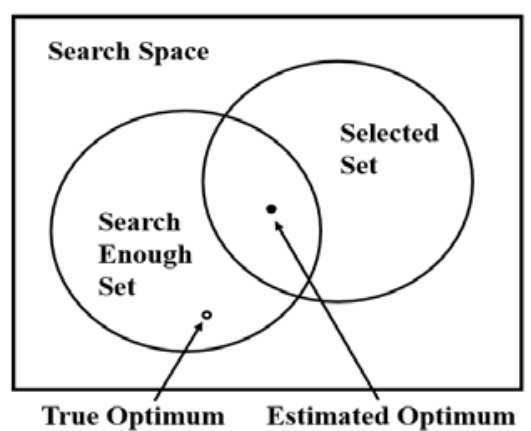

Fig. 1. Schematic diagram of the set of solutions in $\mathrm{OO}$

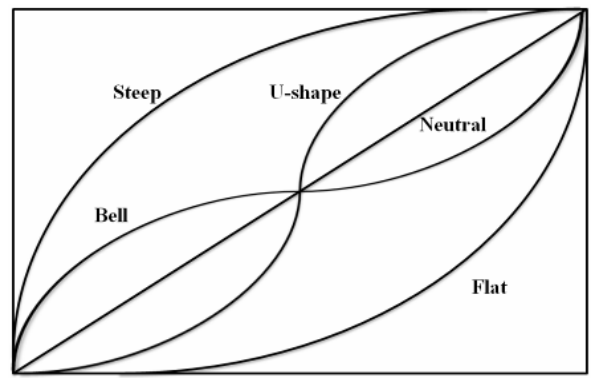

Fig. 2. Five types of ordered performance curves (OPCs).

solution is sorted by the evaluated results of the crude model, and a certain number of top performance solutions are selected to be evaluated by the accurate model. The sufficiently good solution is selected according to the accurate evaluation results.

As shown in Fig. 2, five types of order performance curves (OPCs) can be roughly categorized in OO: Flat (many good designs), Steep (many bad designs), Bell (many mediocre designs), U-shape (either good or bad designs) and Neutral (uniformly distributed designs). The shape of the OPCs determines the number in the set to perform the accurate evaluation [20].

\subsection{Crude evaluation model used for capacity credit}

According to Eq. (3), the capacity credit of wind power $\lambda$ is neither linear nor analytical and thus is difficult to compute because the weight of different hours of wind power output $k_{t}$ is a function of $\lambda$. Here, we use the average output of wind power to approximate $\lambda C_{\mathrm{w}}$ :

$$
k_{t}=\frac{f_{R F}\left(d_{t}-p_{t}+\sum_{t=1}^{T} P_{t}\right)-f_{R F}\left(d_{t}\right)}{\sum_{t=1}^{T} P_{t}-P_{t}}
$$

\subsection{Procedures of solving the wind farm planning problem using $\mathrm{OO}$ theory}

The procedure of solving the proposed wind farm planning problem based on $\mathrm{OO}$ theory is shown as Fig. 3.

Detailed procedures are listed below:

Step I: Sample the value of each $x_{i, a}$ and test if the solution meets the constraints. If the solution does meet the constraints, put the decision into the feasible solution set. Get enough feasible solutions (i.e., 10000), and then go to step II.

Step II: Evaluate the feasible solution set using the crude model in Eq. (3). Sort the feasible solutions by the objective value of the crude model.

Step III: According to the shape of the accumulated curve of the objective values (ordered performance curves, OPCs), decide the number in the set for which to perform 


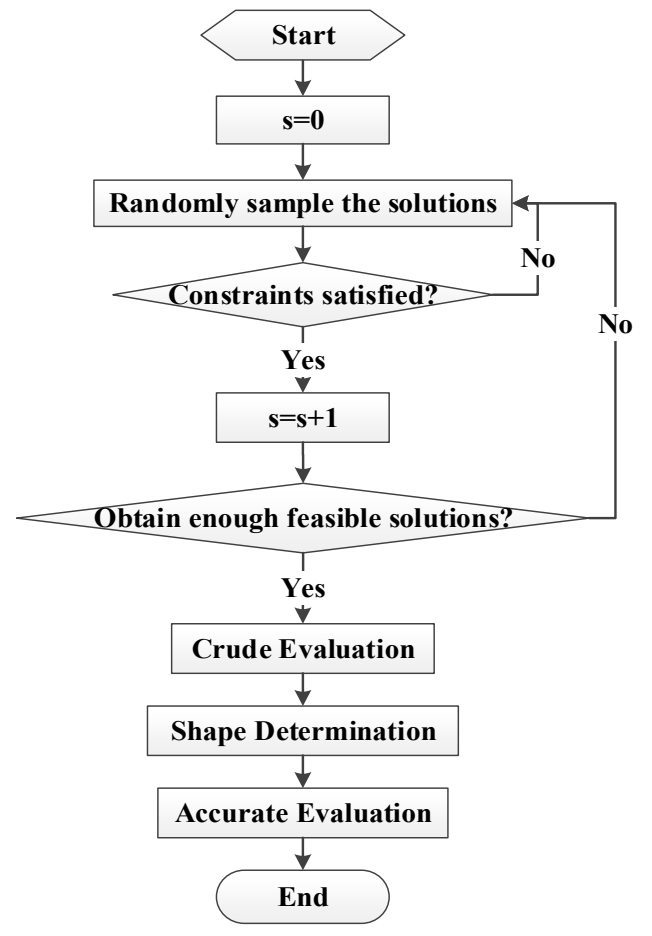

Fig. 3. OO based procedures of wind farm planning theory

the accurate evaluation. The shape of the OPC consists of a Bell shape (as the Case Study will show), so that the formulation of the solution is $M_{k, g}$.

Step IV: Pick the top $M_{k, g}$ solutions in the crude model and evaluate each solution using the accurate model (Eq. (4)). Choose the best solution as the final solution.

\section{Illustrative Example}

\subsection{Data and settings}

The proposed model was tested using a modified IEEE RTS-79 system. Generator data and load were extracted from [27]. Twenty candidate wind farms with $100 \mathrm{MW}$ each were introduced in the planning model and were divided into groups of five. The wind capacity factors in different groups were different and are shown in Fig. 2. The wind farms $1 \sim 5$ (in zone 1) had the highest capacity factor while wind farms 16 20 (in zone 4) had the lowest capacity factor. The correlation coefficients of wind speed among the different zones were set as shown in Table 1. The wind power outputs in the same zone were assumed to be highly correlated with a coefficient of 0.99 . In the actual situation, the spatial correlation among the different wind power zones could be calculated according to the historical wind speed measured by anemometer towers. The yearly outputs of each wind farm were simulated using the method in [28]. The parameters in the planning model were set as shown in Table 2.

In the case study, we consider a five-year wind farm

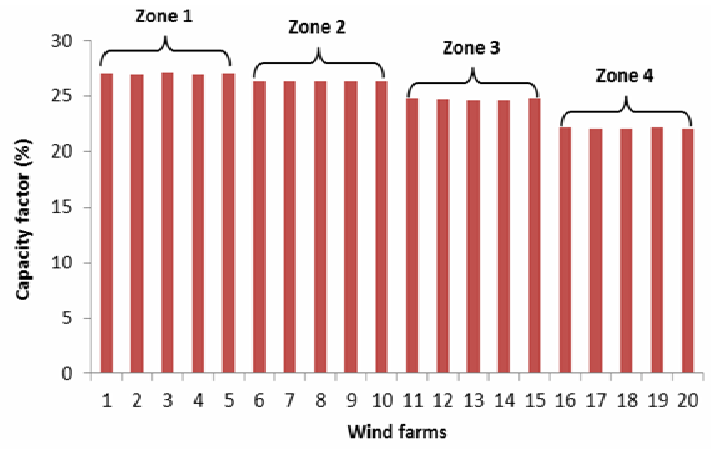

Fig. 4. The capacity factor for candidate wind farms

Table 1. Linear correlation coefficient among different zones

\begin{tabular}{c|c|c|c|c}
\hline & Zone 1 & Zone 2 & Zone 3 & Zone 4 \\
\hline Zone 1 & 1.00 & 0.85 & 0.70 & 0.16 \\
\hline Zone 2 & 0.85 & 1.00 & 0.81 & 0.18 \\
\hline Zone 3 & 0.70 & 0.81 & 1.00 & 0.22 \\
\hline Zone 4 & 0.16 & 0.18 & 0.22 & 1.00 \\
\hline
\end{tabular}

Table 2. Settings for the parameters in the case study

\begin{tabular}{c|c}
\hline Parameter & Value \\
\hline$r$ & $8 \%$ \\
\hline$K_{\mathrm{w}}$ & 15 years \\
\hline$K_{\mathrm{c}}$ & 25 years \\
\hline$\left(\mathrm{P} / \mathrm{A}, r, K_{\mathrm{w}}\right)$ & 0.1082 \\
\hline$\left(\mathrm{P} / \mathrm{A}, r, K_{\mathrm{c}}\right)$ & 0.0867 \\
\hline$I_{\mathrm{w}, i}, I=1,2, \ldots, N$ & $1193 \$ / \mathrm{kW}$ \\
\hline$I_{\mathrm{c}, i}, I=1,2, \ldots, N$ & $645 \$ \mathrm{~kW}$ \\
\hline$O_{i}, I=1,2, \ldots, N$ & $24.03 \$$ \\
\hline$\rho_{i}, I=1,2, \ldots, N$ & $48.4 \$ / \mathrm{MWh}$ \\
\hline$C_{\mathrm{w}, i}, I=1,2, \ldots, N$ & $100 \mathrm{MW}$ \\
\hline$M_{k, g}$ & 139 \\
\hline
\end{tabular}

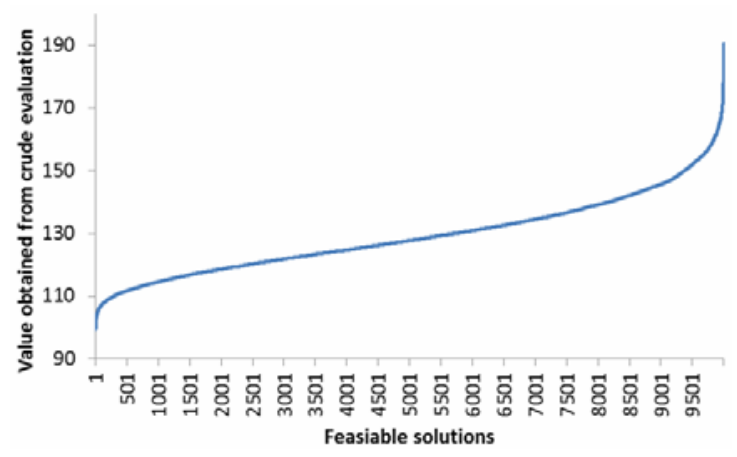

Fig. 5. The OPC of the proposed planning model

planning decision. The capacity requirement is $300 \mathrm{MW}$ in the first year and increases by 300 MW for each year. The total wind farm capacity requirement is $1500 \mathrm{MW}$ in the $5^{\text {th }}$ year.

\subsection{OPC shape determination}

Fig. 5 shows the OPC obtained in step II of the proposed approach. According to the classification of the OPCs in 
the OO theory [26], the OPC shown in Fig. 5 belongs to the Bell shaped OPCs.

\subsection{Number of solutions for accurate evaluation}

The formulation of $M_{k, g}$ is therefore chosen under the Bell OPC category.

$$
M_{k, g}=\left[e^{8.1998} k^{1.9164} g^{-2.0250}+10\right]=139
$$

Here $[\bullet]$ denotes the rounding of the number, and $g$ and $k$ are the parameters of confidence and optimality. We chose $g=10, k=1$, meaning that we wished to find one or more decisions that ranked within the best 10 decisions in the feasible solution set, with the confidence level of $95 \%$.

\subsection{Solutions}

To demonstrate how the capacity benefit of wind power impacts the planning, three cases were conducted as
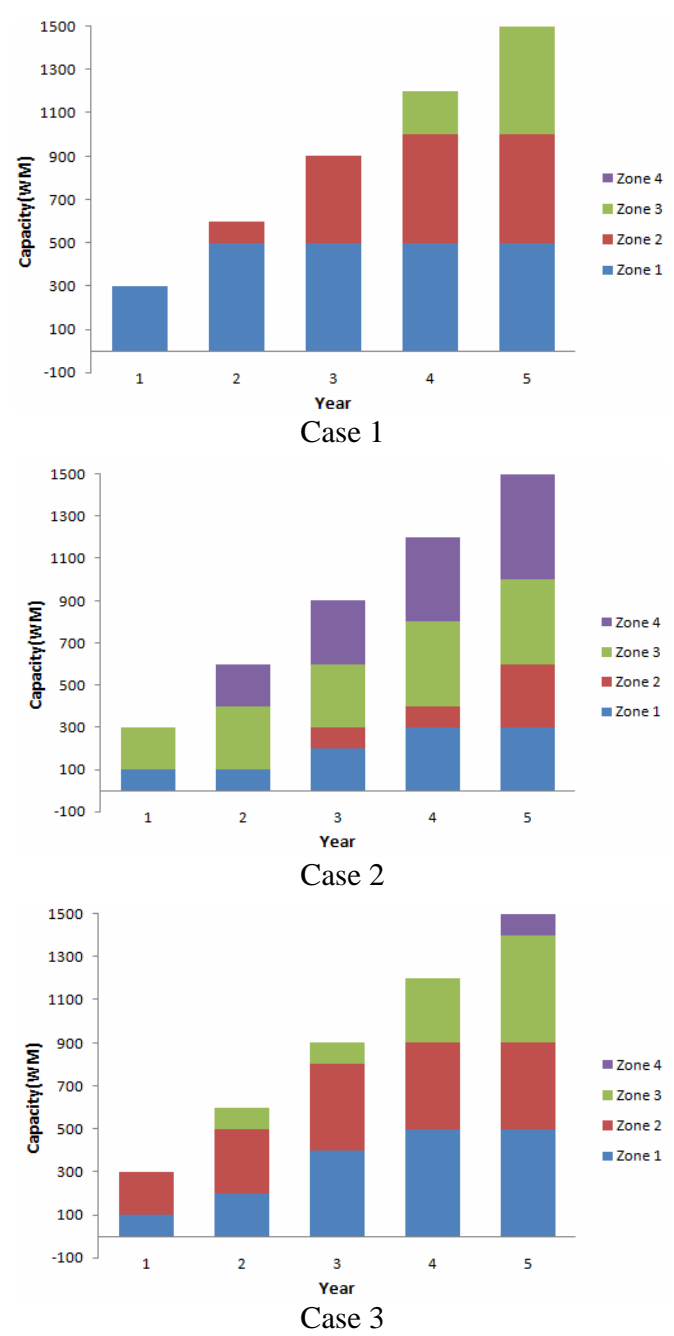

Fig. 6. The planned wind farm capacity in each zone for the three cases follows, and the optimal planning result as listed in Fig. 6.

Case 1: Considering only the energy benefit.

Case 2: Considering only the capacity benefit.

Case 3: Considering both capacity and energy benefit.

In Fig. 6, the planned wind power capacity in each zone for each year is compared to illustrate the difference in planning decisions under different objectives. The capacity factor and the capacity credit associated with each planning decision are listed in Table 3.

In Case 1, when considering only the energy benefit as most planning models do, the planning decision tends to give priority to the wind farms with higher capacity factors.

In Case 2, when considering only the capacity benefit, the planning decision tends to disperse the wind farm investment to obtain a higher capacity credit. In this case, the wind farms that have a higher capacity factor (in zone 1 and zone 2) are not fully planned because the correlation between these two zones is strong and would result in a smaller capacity credit. By contrast, the wind farms in zone 4 that have the lowest capacity factors are fully planned because the correlation with the wind farms in other zones is weak.

Case 3 has a neutralized result of case 1 and 2, where the wind farms are planned to be dispersed, while giving the priority to the wind farm with the higher capacity factor. The results suggest that the proposed model can effectively take into consideration the benefit of the capacity value of wind farm in planning decisions.

The capital and O\&M costs, energy benefit, and capacity benefit for different cases are shown in Table 4. The total cost can be minimized by considering both the energy benefit and the capacity benefit.

Table 3. Average capacity factor and capacity credit for different cases.

\begin{tabular}{|c|c|c|c|c|}
\hline & \multirow{2}{*}{ Year } & \multicolumn{3}{|c|}{ Result (\%) } \\
\hline & & Case 1 & Case 2 & Case 3 \\
\hline \multirow{5}{*}{$\begin{array}{c}\text { Capacity } \\
\text { Factor }\end{array}$} & 1 & $27.07 \%$ & $25.48 \%$ & $26.65 \%$ \\
\hline & 2 & $26.92 \%$ & $24.65 \%$ & $26.32 \%$ \\
\hline & 3 & $26.76 \%$ & $24.56 \%$ & $26.49 \%$ \\
\hline & 4 & $26.39 \%$ & $24.73 \%$ & $26.23 \%$ \\
\hline & 5 & $26.05 \%$ & $24.82 \%$ & $25.76 \%$ \\
\hline \multirow{5}{*}{$\begin{array}{c}\text { Capacity } \\
\text { Credit }\end{array}$} & 1 & $21.29 \%$ & $22.47 \%$ & $21.26 \%$ \\
\hline & 2 & $15.76 \%$ & $17.80 \%$ & $16.55 \%$ \\
\hline & 3 & $12.24 \%$ & $14.81 \%$ & $12.62 \%$ \\
\hline & 4 & $10.38 \%$ & $12.45 \%$ & $10.61 \%$ \\
\hline & 5 & $8.99 \%$ & $10.72 \%$ & $9.54 \%$ \\
\hline
\end{tabular}

Table 4. Costs and benefits for different cases.

\begin{tabular}{c|c|c|c|c}
\hline & $\begin{array}{c}\text { Capital and } \\
\text { O\&M Costs (\$) }\end{array}$ & $\begin{array}{c}\text { Energy } \\
\text { Benefit (\$) }\end{array}$ & $\begin{array}{c}\text { Capacity } \\
\text { Benefit (\$) }\end{array}$ & $\begin{array}{c}\text { Total } \\
\text { Cost (\$) }\end{array}$ \\
\hline Case 1 & 56.319 & 41.330 & 1.552 & 13.437 \\
\hline Case 2 & 56.319 & 38.418 & 2.922 & 14.980 \\
\hline Case 3 & 56.319 & 40.858 & 2.569 & 12.892 \\
\hline
\end{tabular}




\subsection{Comparison with Genetic Algorithm (GA)}

A GA method proposed by the Genetic Algorithm Solver in MATLAB is employed to solve the same wind farm planning optimization model as Case 3 . The key parameters of the GA model are listed below:

Population Size: 300

Generations: 100

Crossover Probability: 0.80

Mutation Probability: 0.02

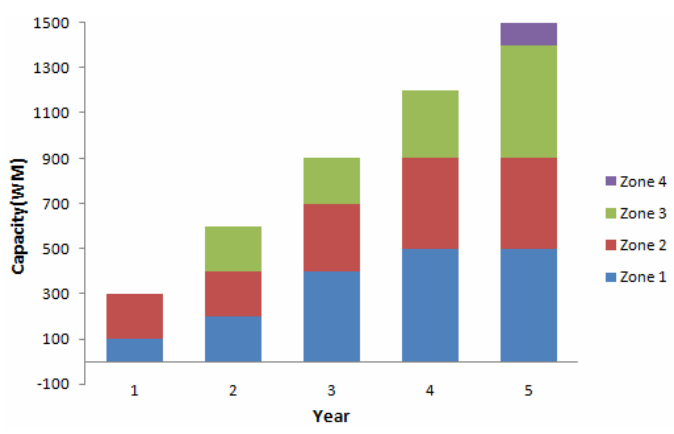

Fig. 7. Optimal solution for GA

Fig. 7 shows the optimal solution for GA. Compared with the results shown in Fig. 6 for Case 3, there is little difference in the second and third planning years. The optimal value of GA is $\$ 12.994$ million, which is a little larger than the optimal value of $\mathrm{OO}$ (\$12.892 million). Additionally, the convergence of GA is not well performed because each simulation gets different results.

\section{Application to Ningxia Provincial Power Grid}

\subsection{Wind power planning in ningxia province}

In this section, an application of the proposed approach is conducted in Ningxia Province, China, which is rich in wind power.

There are several wind farms distributed in six zones, named Qingtongxia(QT), Taiyangshan (TY), Mahuangshan (MH), Xiangshan (XS), Jiucaishan (JC), and Magaozhuang (MG). Through the analysis of historical data in Ningxia, the Weibull parameters of different wind zones and correlation coefficients among them can be obtained (shown in Table 5 and Table 6, respectively). Table 5 shows that $\mathrm{MH}$ is the richest in wind resources, while TY is the

Table 5. Weibull parameters of the six wind zones

\begin{tabular}{c|c|c}
\hline & c & $\mathrm{k}$ \\
\hline QT & 7.6636 & 2.2117 \\
\hline TY & 7.5742 & 2.3222 \\
\hline MH & 7.7237 & 2.1375 \\
\hline XS & 7.6577 & 2.2190 \\
\hline JC & 7.6489 & 2.2299 \\
\hline MG & 7.6291 & 2.2175 \\
\hline
\end{tabular}

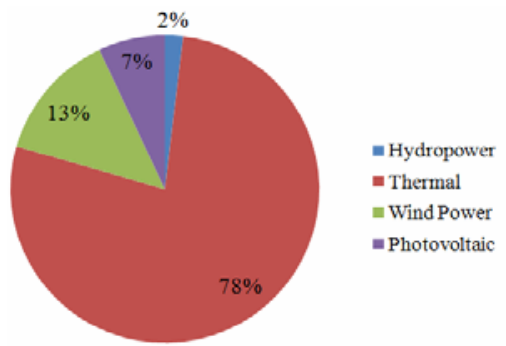

Fig. 8. Capacity of different forms of power of Ningxia in 2013

Table 6. Linear correlation coefficient among six zones

\begin{tabular}{c|c|c|c|c|c|c}
\hline & QT & TY & MH & XS & JC & MG \\
\hline QT & 1 & 0.6473 & 0.5754 & 0.6101 & 0.5121 & 0.5920 \\
\hline TY & 0.6473 & 1 & 0.7523 & 0.5761 & 0.5593 & 0.7090 \\
\hline MH & 0.5754 & 0.7523 & 1 & 0.5281 & 0.5521 & 0.7926 \\
\hline XS & 0.6101 & 0.5761 & 0.5281 & 1 & 0.6187 & 0.6121 \\
\hline JC & 0.5121 & 0.5593 & 0.5521 & 0.6187 & 1 & 0.6534 \\
\hline MG & 0.5920 & 0.7090 & 0.7926 & 0.6121 & 0.6534 & 1 \\
\hline
\end{tabular}
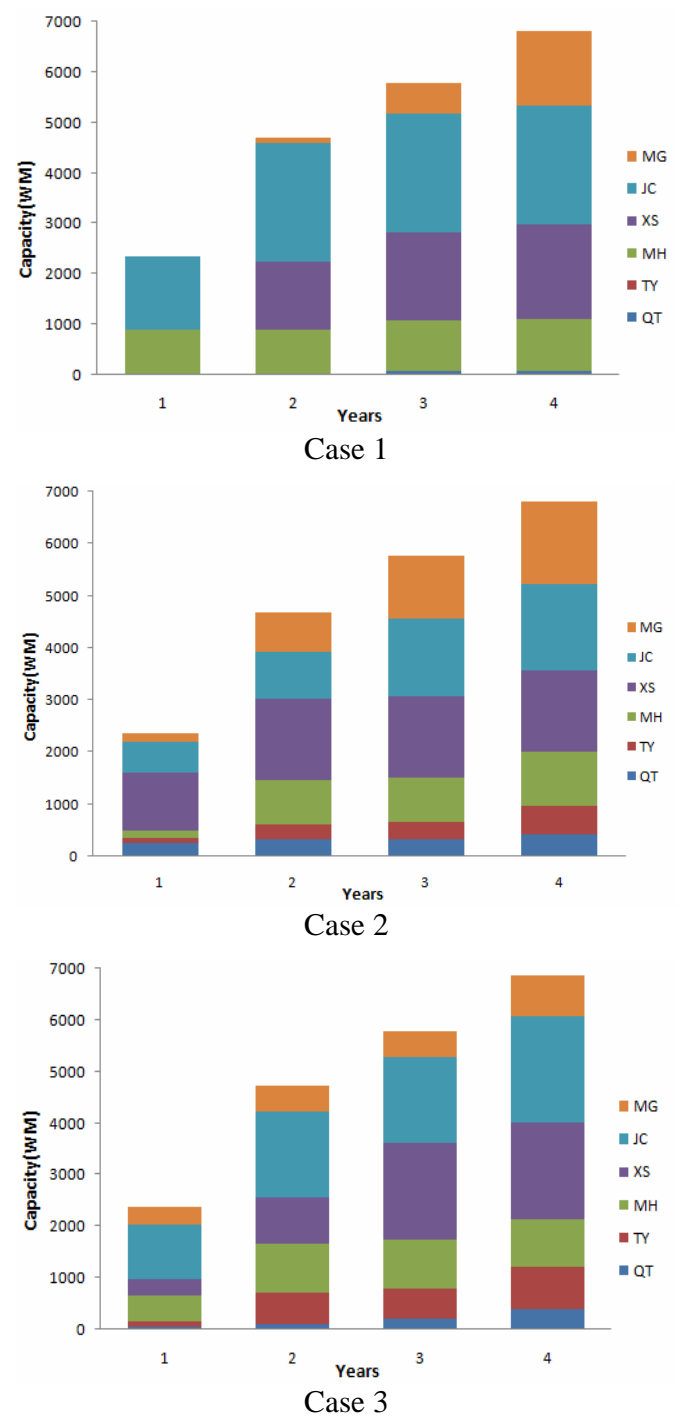

Fig. 9. The planned wind farm capacity in Ningxia for the three cases 
poorest. The correlation among the six wind zones in Ningxia is at a relatively higher level, different from the illustrative example in Section 5

As shown in Fig. 8, by the end of 2013, the installed capacity of hydropower, thermal power, wind power and photovoltaic power in Ningxia is 426 MW, 17313 MW, 3018 MW and $1561 \mathrm{MW}$, respectively. The power consumption of the whole society in Ningxia is 8.112 billion $\mathrm{kWh}$ in 2013, and the maximum electricity load had reached 11260 MW.

We consider a four-year (from 2014 to 2017) wind farm planning decision. The capacity requirement in each year is $2334 \mathrm{MW}, 4668 \mathrm{MW}, 5742 \mathrm{MW}$, and $6815 \mathrm{MW}$. The maximum planning capacity of each of the six wind zones in Ningxia is 520 MW, 1950 MW, 1045 MW, 1872 MW, $2350 \mathrm{MW}$, and $2450 \mathrm{WM}$.

\subsection{Results and discussion}

The same three cases as shown in Fig. 6 are used in the wind power planning in Ningxia, and the optimal solution is shown in Fig. 9. Fig. 9 illustrates that the zones with rich wind power resources ( $\mathrm{MH}$ and $\mathrm{JC}$ ) has the priority to be invested, while the zones with lower correlation (XS and $\mathrm{MH})$ have the priority to be invested to maximize the capacity benefit as well.

\section{Conclusions}

Large-scale clustered wind farms could contribute to generation adequacy, so the capacity benefit of wind power should be considered in wind farm planning. This paper proposes a wind farm planning model that takes into account the capacity benefit of wind farms. The $\mathrm{OO}$ technique is used to solve the problem. The wide spread of wind farms with less correlation would result in a higher capacity credit. Considering only the energy benefit may mislead the investment and result in a lower wind power capacity credit. An illustrative example of a modified IEEE RTS 79 system and a case study in Ningxia shows that the capacity benefit of the wind power should be considered apart from the energy benefit in wind power planning.

\section{Acknowledgements}

This work was supported in part by National Natural Science Foundation of China (No.51325702, 51307092), China Postdoctoral Science Foundation funded project (2013T60113) and technical projects of State Grid.

\section{References}

[1] Chinese Wind Energy Association (CWEA). 2013
Statistics of the installed capacity of wind power in China.http://windpower.ofweek.com/2014-03/ART330002-8420-28792372.html.

[2] Energy Resource Institute of China's National Reform and Development Commission. Technology Roadmaps: China Wind Energy Development Roadmap 2050. 2011.

https://www.iea.org/publications/freepublications/pu blication/china_wind.pdf.

[3] S. Zhang, and X. Li, "Large scale wind power integration in China: Analysis from a policy perspective," Renewable and Sustainable Energy Reviews, vol. 16, no. 2, pp. 1110-1115, Feb. 2012.

[4] M. Xu, and X. Zhuan, "Optimal planning for wind power capacity in an electric power system,” Renewable Energy, vol. 53, pp. 280-286, May. 2013.

[5] L. Baringo, and A.J. Conejo, "Risk-constrained multistage wind power investment," IEEE Trans. Power Systems, vol. 28, no. 1, pp. 401-411, Feb. 2013.

[6] L. Baringo, and A. J. Conejo, "Transmission and wind power investment,” IEEE Trans. Power Systems, vol. 27, no. 2, pp. 885-893, May. 2012.

[7] M. Nick, G.H. Riahy, S. H. Hosseinian, and F. Fallahi, "Wind power optimal capacity allocation to remote areas taking into account transmission connection requirements," IET Renewable Power Generation, vol. 5, no. 5, pp. 347-355, Sep. 2011.

[8] S. Liu, J. Jian, Y. Wang, and J. Liang, "A robust optimization approach to wind farm diversification," International Journal of Electrical Power \& Energy Systems, vol. 53, pp. 409-415, Dec. 2013.

[9] A. Keane, M. Milligan, C.J. Dent, B. Hasche, C. D’Annunzio, K. Dragoon, H. Holttinen, N. Samaan, L. Soder, and M. O’Malley, "Capacity Value of Wind Power,” IEEE Trans. Power Systems vol. 26, no. 2, pp. 564-572, May. 2011.

[10] N. Zhang, C. Kang, Z. Chen, Y. Zhou, J. Huang, and W. Xi, "Wind power credible capacity evaluation model based on sequence operation," Proceedings of the CSEE, vol. 31, no. 25, pp.1-9, 2011.

[11] X. Lu, M.B. McElroy, C.P. Nielsen, X. Chen, and J. Huang, "Optimal integration of offshore wind power for a steadier, environmentally friendlier, supply of electricity in China,” Energy Policy, vol. 62, pp. 131138, Nov. 2013.

[12] H Li, D F Cai, Y Li. "Probabilistic Assessment of Voltage Stability Margin in Presence of Wind Speed Correlation," Journal of Electrical Engineering and Technology, vol. 8, no. 6, pp. 719-728, Jul. 2013.

[13] Y Li, K Xie, B Hu. "Copula-ARMA model for multivariate wind speed and its applications in reliability assessment of generating systems," Journal of Electrical Engineering and Technology, vol. 8, no. 3, pp. 421-427, May. 2013.

[14] N. Zhang, C. Kang, Q. Xu, H. Li, J. Xiao, Z. Wang, R. Shi, and S. Wang "Clustered wind farms planning 
considering the capacity credit using ordinal optimization technique," in International Conference on Wind energy Grid-Adaptive Technologies 2014 (WeGAT 2014), Jeju, Korea,Oct.2014.

[15] M. Amelin, "Comparison of capacity credit calculation methods for conventional power plants and wind power," IEEE Trans. Power Systems, vol. 24, no. 2, pp. 685-691, May. 2009.

[16] N. Zhang, C. Kang, Q. Xu, C. Jiang, Z. Chen, and J. Liu, "Modelling and Simulating the Spatio-Temporal Correlations of Clustered Wind Power Using Copula,” Journal of Electrical Engineering and Technology, vol. 8, no. 6, pp. 1615-1625, Nov. 2013.

[17] H. Wang, Z. Lu, and S. Zhou, "Research on the capacity credit of wind energy resources," Proceedings of the CSEE, vol. 25, no. 10, pp.103-106, May. 2005.

[18] R. Karki, P. Hu, and R. Billinton, "A simplified wind power generation model for reliability evaluation," IEEE Trans. Energy Conversion. vol. 21, no. 2, pp. 533-540, Jun. 2006.

[19] N. Zhang, C. Kang, D. S. Kirschen, and X. Qing, "Rigorous model for evaluating wind power capacity credit,” IET Renewable Power Generation, vol. 7, no. 5, pp. 504-513, Sep. 2013.

[20] Y. Ho, Q. Zhao, and Q. Jia, “Ordinal Optimization Soft Optimization for Hard Problems": Springer, 2007.

[21] X. Guan, Y. C. Ho, and F. Lai, "An ordinal optimization based bidding strategy for electric power suppliers in the daily energy market," IEEE Trans. Power Systems, vol. 16, no. 4, pp. 788-797, Nov. 2001.

[22] X. Guan, Y. C. Ho, F. Lai, “An ordinal optimization based bidding strategy for electric power suppliers in the daily energy market," IEEE Trans. Power Systems vol. 16, no. 4, pp. 788-797, Nov. 2001

[23] S.Y. Lin, Y.C. Ho, and F. Lai, “An ordinal optimization theory-based algorithm for solving the optimal power flow problem with discrete control variables," IEEE Trans. Power Systems vol. 19, no. 1, pp. 276286, Feb. 2004

[24] Y. C. Chang, R. F. Chang, T. Y. Hsiao, and C. N. Lu, , "Transmission system loadability enhancement study by ordinal optimization method," IEEE Trans. Power Systems vol. 26, no. 1, pp. 451-459, Feb 2011

[25] R. A. Jabr, B. C. Pal, "Ordinal optimization approach for locating and sizing of distributed generation," IET generation, transmission \& distribution, vol. 3, no. 8, pp. 713-723, Aug. 2009.

[26] T. W. E. Lau and Y. C. Ho, "Universal alignment probabilities and subset selection for ordinal optimization," Journal of Optimization Theory and Applications, vol. 93, no. 3, pp. 445-489, Jun. 1997.

[27] Probability Methods Subcommittee, "IEEE reliability test system," IEEE Trans. Power Apparatus and Systems, vol. PAS-98, no. 6, pp. 2047-2054, Nov. 1979.

[28] N. Zhang, C. Kang, C. Duan, X. Tang, J. Huang, Z. Lu, W. Wang, and J. Qi, "Simulation methodology of multiple wind farms operation considering wind speed correlation," International Journal of Power and Energy Systems, vol. 30, no. 4, pp. 264-173, Apr. 2010.

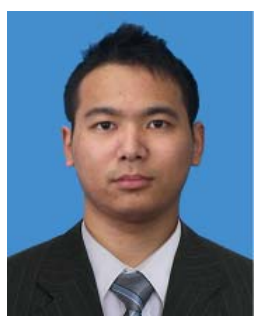

Yi Wang He received his bachelor's degree from the Department of Electrical Engineering of Huazhong University of Science and Technology in China in 2014. He is currently working towards the Ph.D. in Tsinghua University. His research interests include wind power planning, demand response, and big data application in power system.

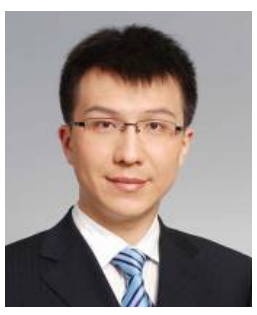

Ning Zhang He received both B.S. and Ph.D. from the Electrical Engineering Department of Tsinghua University in China in 2007 and 2012, respectively. He is now a Post-doctor in Tsinghua University. His research interests include stochastic characteristic analysis and simulation of renewable energy, power system planning and scheduling with renewable energy.

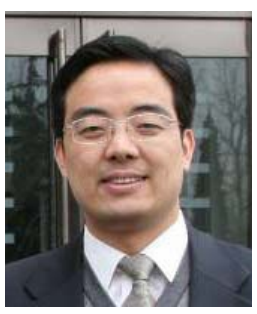

Chongqing Kang $\mathrm{He}$ received his Ph.D. from the Dept. of Electrical Engineering of Tsinghua University in 1997. He is now a Professor at the same university. His research interests include low-carbon electricity, power system planning, renewable energy and power system load forecasting.

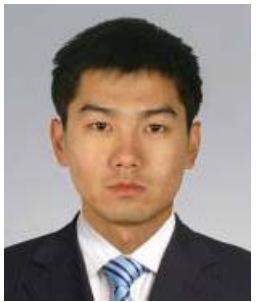

Qianyao Xu He received his bachelor's degree from the Department of Electrical Engineering of Tsinghua University in China in 2010. He is currently working towards the Ph.D. in the same University. His research interests is wind power planning and accommodation assessment. 
Hui Li He received both B.S. and Ms.c from the Electrical Engineering Department of Tsinghua University in China in 2002. He is now the director of the transmission network planning center of State Power Economic Research Institute.

Jinyu Xiao He received both B.S. and Ph.D. from the Electrical Engineering Department of Tsinghua University in China in 2004. He is now the deputy director of the transmission network planning center of State Power Economic Research Institute.

Zhidong Wang He received Ms.c from the School of Electrical \& Electronic Engineering of North China Electric Power University in China in 2007. He is now a senior engineer in the transmission network planning center of State Power Economic Research Institute.

Rui Shi He received Ms.c from the School of Electrical \& Electronic Engineering of The University of Manchester in UK in 2012. He is now an engineer in the transmission network planning center of State Power Economic Research Institute.

Shuai Wang He received Ms.c from the Electrical Engineering School of Xi'An Jiaotong University in China in 2006. He is now a senior engineer in the transmission network planning center of State Power Economic Research Institute. 